\title{
Numerical simulation and experimental study of the dynamic characteristics of an impact testing machine
}

\author{
Z. F. Deng, F. J. Zhang \& G. Chen \\ Institute of Systems Engineering, CAEP, China
}

\begin{abstract}
For detecting dynamic parameters of apparatus, some specified impact experiments must be carried out on impact testing machines. The various requirements of acceleration peak and impulse duration are demanded for the impact testing machines with different functions. The material properties and geometrical configuration of the striker and target of impact testing machines are important factors for the detected acceleration responses. In the present work, the experiments and numerical simulations are carried out to investigate the influence of the geometrical configuration of the striker and material properties of the target on acceleration curves. The analysis indicates that the acceleration curve greatly depended on the geometrical configuration of the striker and material properties of the target. Analyzing those dynamic response characteristics of impact testing machines is helpful for improving equipment quality.

Keywords: impact testing machine, dynamic response, acceleration curve, acceleration peak, impulse duration, geometrical configuration, material properties.
\end{abstract}

\section{Introduction}

For detecting the dynamic parameters of apparatus, some specified impact experiments must be carried out on impact testing machines. However, one piece of equipment can't finish all impact experiments, and experiments with different functions need various requirements for acceleration peak and impulse duration. Generally, acceleration peak and impulse duration are contrary to each other, which means that the big acceleration peak always accompanies short pulse 
duration, and the small acceleration peak always accompanies long pulse duration in equivalent intensity impact experiments.

There are lots of impact testing machines at present. The means to implement impact process varies greatly, such as drop test mode, pendulum impact test mode, etc. The pendulum impact test experiment is a classical method to estimate impact toughness of samples. The pendulum impact testing machine is widely used for its simple structures, convenient operations, low cost, and may obtain stable and repeatability experimental results.

In the present paper the dynamic response of a pendulum impact testing machine is studied. In experiments, the stress wave produced by the striker impacting the target, and then acts on the sample to estimate the dynamic characteristics of samples. So the striker and the target are the crucial factors of the impact strength. In addition, the striker and the target may produce accumulated plastic deformation, and the local materials become hardened when the equipment is used for a long time/frequency (called old equipment). So the experimental results may not be as accurate as before.

Many experiments and numerical simulations of the pendulum impact testing machine are carried out to study the equipment dynamic characteristics and mechanism. In present paper the striker and target with different service condition and material are studied to understand its influence on the dynamic response characteristics of the equipment, and the research is helpful for improving equipment quality.

\section{A brief introduction of pendulum impact testing machine}

Figure 1 shows the mechanism structural scheme of a pendulum impact testing machine.

The major components of the equipment include swing arm, pendulum, striker, target, inert unit. The inert unit is used for scaling impact load, and its
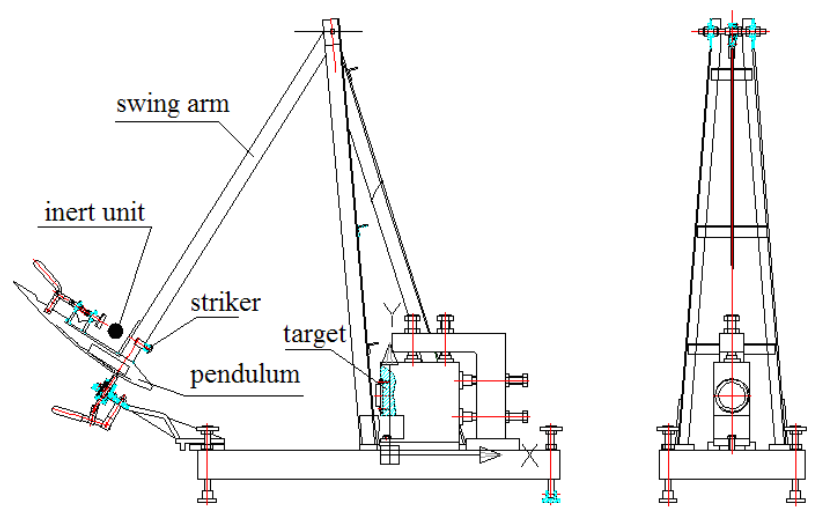

Figure 1: The mechanism structural scheme of the pendulum impact testing machine. 
state changes when impact strength achieves a certain value. In experiments, the swing arm is released at a predetermined height, then swings to the low position. The angular velocity increases to maximum when the rotation parts gravity center achieve the lowest position, then the striker impacts the target. The impact load acts on the inert unit, causing the inert unit state changes.

\section{Experimental results}

The test system for measure impact accelerations includes acceleration sensor, charge amplifier, and digital oscilloscope. The calibrated inert unit placed the inert unit position. When the swing arm releases at different height and the striker impacts the target, the impact accelerations are measured by acceleration sensors, and normalized amplified by charge amplifier. Then the impulse is recorded by digital oscilloscope.

There are three measuring points lying on the equipment, which are defined L, $\mathrm{R}$ and $\mathrm{M}$ (shown in Figure 2). The measuring points $\mathrm{L}$ and $\mathrm{R}$ locate on the mounting plate which front of inert unit, and the measuring point $\mathrm{M}$ locates on the fixed plate which far off the back of inert unit. Three acceleration sensors are placed at the measuring point's position.

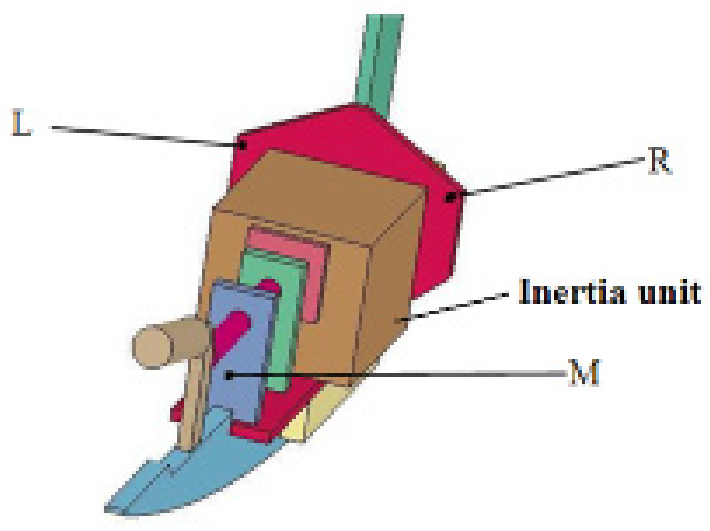

Figure 2: Measuring points' layout.

Many experiments are finished on the pendulum impact testing machine. The typical acceleration curves are shown in Figure 3. Figure 3(a) shows the acceleration curves obtained at different initial released height with new striker and target (called new equipment). Figure 3(b) shows the acceleration response of measuring points $\mathrm{L}$ and $\mathrm{R}$ at a certain initial release height $(14.5 \mathrm{~g}$. $\mathrm{g}$ is a calibration to scale the release height of the swing arm. The bigger the previous number, the higher the swing arm is released (with the old equipment). Seen from the figure, the acceleration peak and trend of the two points are nearly identical. The acceleration peak is about $3550 \mathrm{~g}$ ( $\mathrm{g}$ is the gravity acceleration), and the impulse duration is approximately $180 \mu \mathrm{s}$. Other experimental results in 
the same conditions are shown in Figure 3(c), with the acceleration curve of measuring point $\mathrm{M}$.

Figure 3 reveals that the total acceleration trend is similar, and the acceleration peaks increase as the initial release height increase. The acceleration peak and trend of measuring point $\mathrm{L}$ and $\mathrm{R}$ are nearly identical. But the

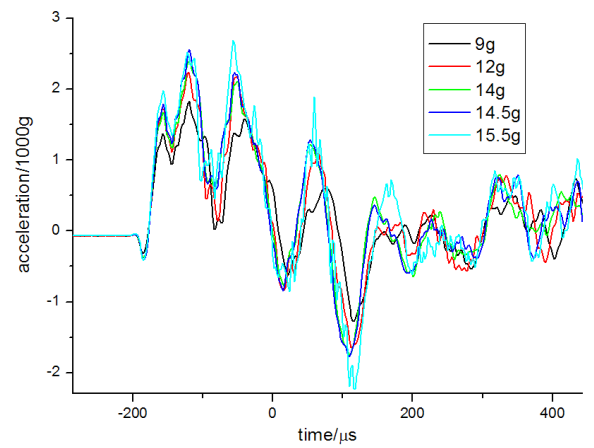

(a)

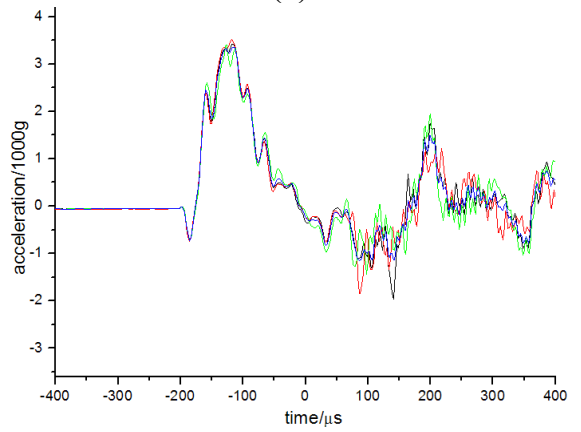

(b)

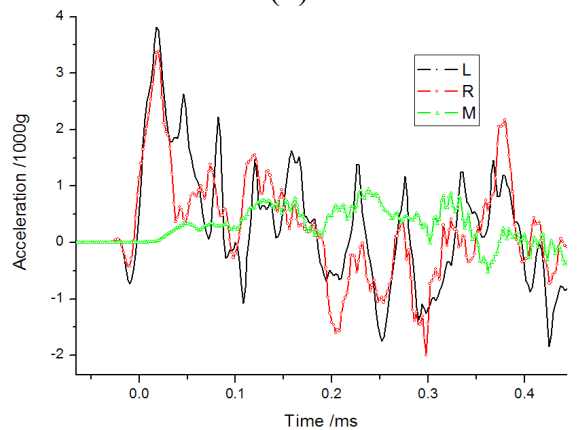

(c)

Figure 3: (a) Acceleration curves obtained at different initial released height with new equipment; (b) acceleration curves of measuring points $\mathrm{L} / \mathrm{R}$ with old equipment (14.5g); (c) Acceleration curves of the three measurement points with old equipment (14.5g). 
acceleration response of measuring point $\mathrm{M}$ is significant different from the previous two. The acceleration peak of measuring point $M$ is about $1 / 4$ of the other two, but its impulse duration is creased to 3 to 4 times about the other two.

\section{Numerical simulation}

\subsection{Finite element model of the pendulum impact testing machine}

Hexahedral solid elements are adopted to simulate the pendulum impact testing machine model. There acceleration sensors and inert unit are simplified by equivalent mass. The target fixed by a set of structures in practice, is simplified by a circular plate. Figure 4 shows the whole finite element model. Major contact interfaces link each other by the method of using same nodes, and the others by adhesion. The striker and the target adopt automatic surface-to-surface contact to simulate the impact process. The boundary conditions are as following: 1) fix the nodes of the top center of swing arm; 2) fix the nodes of the bottom of target; 3 ) set the target bottom and side surface the non- reflecting surfaces.

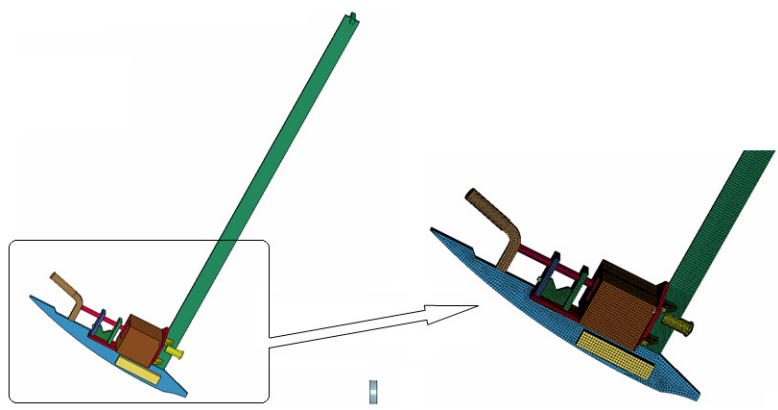

Figure 4: Finite element model of the pendulum impact testing machine.

The original material of the striker and target is chromium alloy. Modulus test experiments reveal the Young's modulus is about 210GPa. Strength test experiments reveal the yield strength is about $2600 \mathrm{MPa}$, and the maximum strength is at least $3800 \mathrm{MPa}$. In numerical simulations, the material model of striker and target is elastoplastic constitutive. The other materials are highstrength steels, and the material model is elastic constitutive, which the density is $7800 \mathrm{~kg} / \mathrm{m}^{3}$ and the Young's modulus is $210 \mathrm{GPa}$.

From the swing arm is released freely to the striker impacts the target; the whole process can be divided into two. The first process is the rotation parts swing freely from initial height to the lowest position by the gravity. The second process is the striker impacts the target. Analysis show that the first process needs 0.5 second, and the second process needs just only 0.001 second. The two times are different in magnitude. For shortening calculation time and simplifying numerical calculation, the two processes are simulated separately. 


\subsection{Numerical simulation of the free swing process}

In the free swing process, the rotation parts are loaded only by gravity. Figure 4 shows the free swing process.
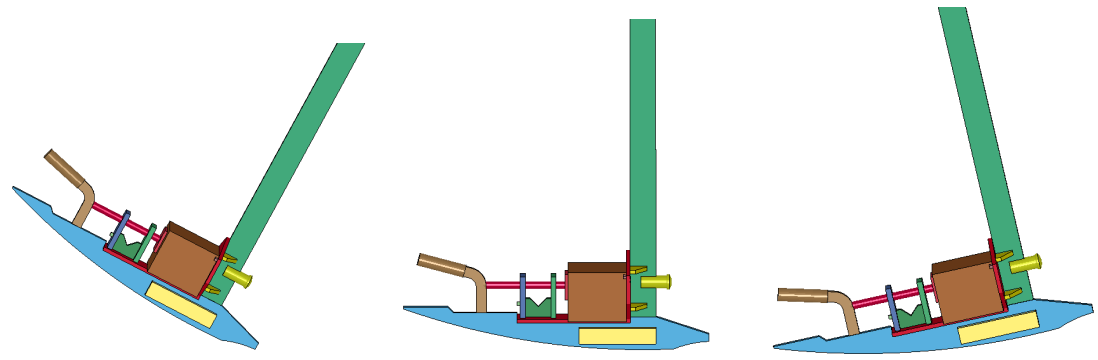

Figure 5: Free swing process of the rotation parts.

The angular velocity history of rotation parts shown in Figure 5, by numerical simulation. The maximum angular velocity of rotation parts is $1.73 \mathrm{rad} / \mathrm{s}$, and at that moment the swing arm is a little right its vertical direction. Another method to know the angular velocity is calculating the rotation parts gravity center at the initial location, then calculating the angular velocity of the rotation parts by means of conversion theory of potential energy and kinetic energy. The results from both methods are consistency.

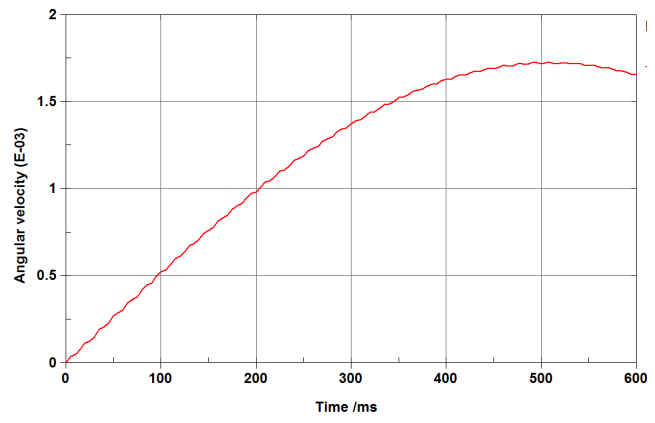

Figure 6: Angular velocity history of rotation parts.

\subsection{Numerical simulation of impact process}

In practice, the swing arm is a little right of its vertical direction when the striker impacts the target. However, in order to establish the finite element model, the initial time of impact process is supposed when swing arm swings to its vertical direction. In this case, the angular velocity of the rotation parts is $1.71 \mathrm{rad} / \mathrm{s}$, which brings about $1 \%$ error comparing the maximum angular velocity. The little error of initial velocity will cause little error on the results. 


\subsubsection{New equipment}

Some numerical simulations for the pendulum impact testing machine with new striker and target are carried out; the corresponding initial height of swing arm is $14.5 \mathrm{~g}$. By observing the results, the maximum stress takes place on the striker and target collision position. The maximum equivalent stress on the striker is about $1300 \mathrm{MPa}$, and on the target is bout $1100 \mathrm{MPa}$. The stress of other regions is too small. Figure 6 shows the acceleration curves of three positions tested by the three acceleration sensors. From the curves, we can see that the accelerations

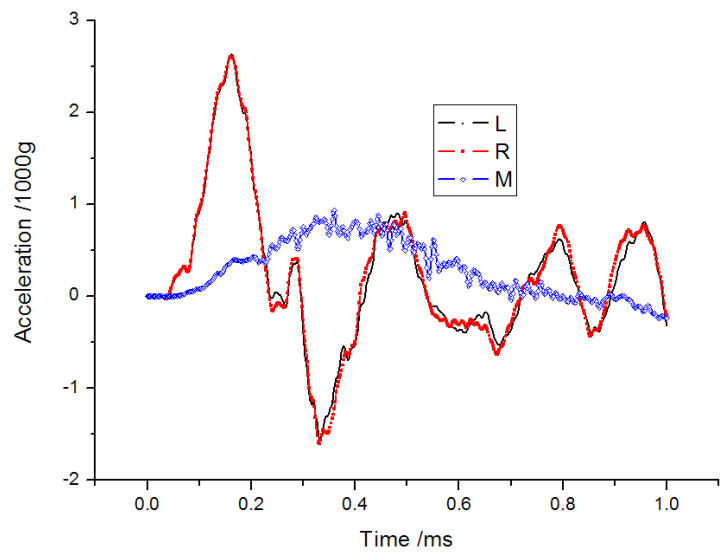

Figure 7: Acceleration curves of three measure points by experiments.

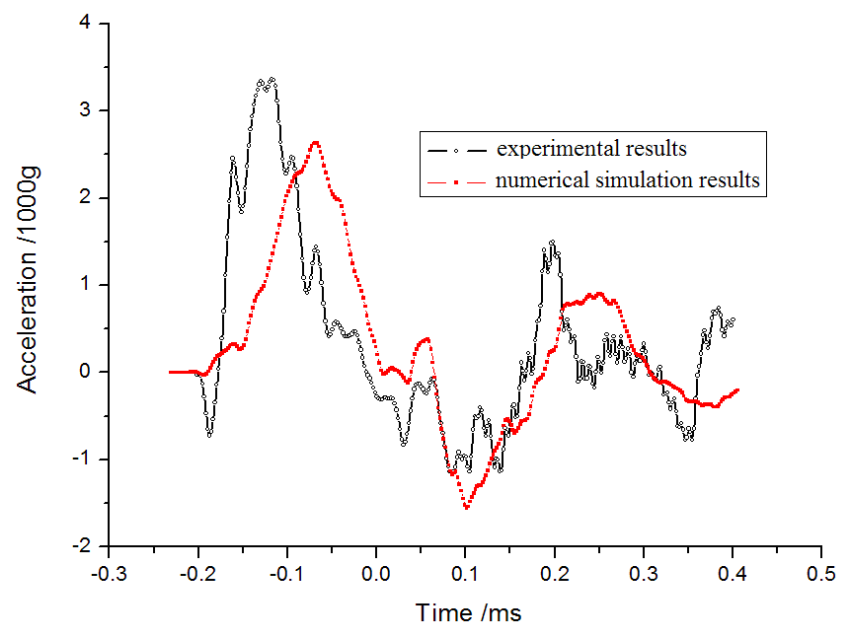

Figure 8: Acceleration curves comparison of measuring points L/R between experimental and numerical simulation results $(14.5 \mathrm{~g})$. 
of measure points $\mathrm{L}$ and $\mathrm{R}$ are perfect consistent with each other, and the acceleration peak is about $2600 \mathrm{~g}$. The acceleration of measure point $\mathrm{M}$ is much gentle and its peak acceleration is about $930 \mathrm{~g}$.

Figure 7 shows the acceleration comparison of measuring points L/R between experimental and numerical simulation results showed. The figure reveals that the trend of two acceleration curves are similar, but the acceleration peak obtained from experiments is higher than numerical simulation about $1 / 3$, and the times of acceleration peaks are also much different.

\subsubsection{Flat head striker}

Due to the acceleration curves of experiments and numerical simulation are much different, we must find out the reasons to make the numerical simulations better reflect the experimental results. After careful observation, we find that the striker gets obvious plastic deformation on old equipment. The striker head isn't a point, and it becomes a small round flat surface. Correspondingly the target gets tiny abrasion on the impacted position. To simulate real state of the old equipment, the striker must revise its head structure to flat head in numerical simulation.

Directly adjusting the mesh nodes on finite elements model, the round head striker changes to flat head striker. The area of flat head assumes approximately $4 \mathrm{~mm}^{2}, 9 \mathrm{~mm}^{2}$ and $18 \mathrm{~mm}^{2}$. Numerical simulation results for those states are shown in Figure 8 (area eq. zero is the new striker).

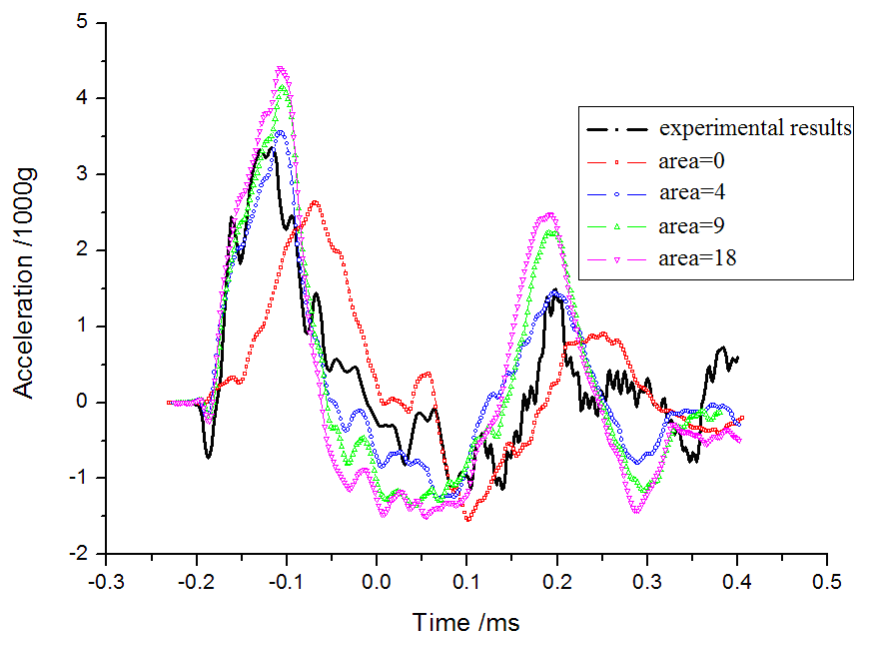

Figure 9: Acceleration curves comparison between numerical simulations of different flat head striker and experiments. 
Figure 8 shows the acceleration curves comparison between numerical simulations of different flat head striker and experiments. It shows that the accelerations response trend of different areas of the flat head striker and experiments is corresponding. With the increase area of flat head striker, the acceleration responses will also increase. Those indicating that with increase time/frequency for the use of striker, the acceleration responses will increase. By totally comparing all acceleration curves, the acceleration peak and peak time of the flat head area equal to $4 \mathrm{~mm}^{2}$ is most close to the experiments curve. These indicate that the equipment really does have abrasion after used for some time/frequency, and the abrasion has much influence on the test results. Those demonstrate the experimental results of old equipment are not correct any more.

The experiment and numerical simulation results show that the old equipment must be replaced to the new striker and target. Alternatively the experimental results must be revised if the old equipment continues to use, or reduce the initial release height of the swing arm, to make the experimental results of old equipment identify to the new equipment.

\subsubsection{Nylon target}

The material of the target is high-strength steel in previous experiments and simulation. In this section, some experiments are carried out with the material of target replacing to nylon, and the material of the striker remains unchanged. Acceleration response curves shown in Figure 9. Seen from the figure, the acceleration peak and trend of three positions are nearly consistent. The acceleration peaks are about $300 \mathrm{~g}$, and the impulse durations are between $1.4 \mathrm{~ms}$ and $1.8 \mathrm{~ms}$. Compared to Figure 3 , the acceleration peak decreased nearly an order of magnitude, but the pulse duration increases almost an order of magnitude. The acceleration curves difference among the three measuring point $\mathrm{L} / \mathrm{R}$ and $\mathrm{M}$ is greatly reduced than before.

In addition, by observing the action of inert unit, the requirements of state changes are decreased (i.e. the swing arm minimal initial release height which can cause the inert unit state changes decreases). Through analysis, we think that the mechanism of inert unit changes its state is a cumulative process for acceleration effect. As long as the acceleration exceeds a certain magnitude, and continues for a period of time, the impact load may cause the inert unit state changes. In previous experiments, the material of striker and target is chromium alloy, and the impact load is intense but short, which may cause the inert unit state changes. In present experiments, the material of striker unchanged but target changed to a softer material, and the impact load is much moderate but longer, which also may cause the inert unit state changes. Both impact loads are the same purpose.

Some numerical simulations are finished with the nylon target. Figure 10 shows the acceleration responses of the three measuring points. Comparing Figure 9 carefully, we find that both acceleration curves have the same acceleration peak and trend. The numerical simulations better reflect the experiments. 


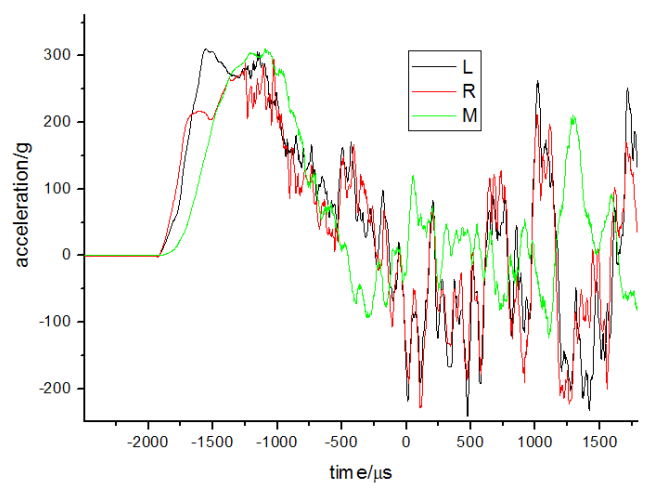

Figure 10: Acceleration responses of experiments with nylon target.

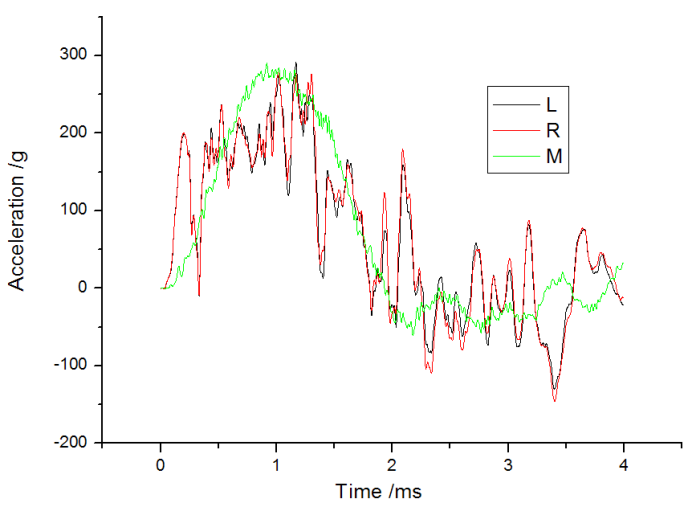

Figure 11: Acceleration responses of numerical simulations with nylon target.

\subsubsection{Dynamic responses analysis of their positions}

For all experimental and numerical simulation results, the measurement points $\mathrm{L}$ and $\mathrm{R}$ are not exactly the same in acceleration response. There is much dispersion. However, the acceleration curves of the measuring points $M$ are always more consistent and stable. Figure 12 shows some acceleration response curves of numerical simulations at different conditions. The figure reveals the similar acceleration response of measuring point $\mathrm{M}$, even if the initial conditions are different greatly.

By observing three positions, measuring points $\mathrm{L}$ and $\mathrm{R}$ are on the edge of the mounting plate where gets relatively lower structural stiffness, and they are closer to the collision region. So both positions are sensitivity to impact loads; while measuring point $\mathrm{M}$ is on the center of fixed plate where gets relatively higher structural stiffness, and it is far from the collision region. So it is stable, and better reflects the dynamic characteristics of entire equipment. Therefore the measuring point $\mathrm{M}$ is the best position for studying the structural dynamic characteristics, and it can be a choice of standard positions for calibration the equipment. 


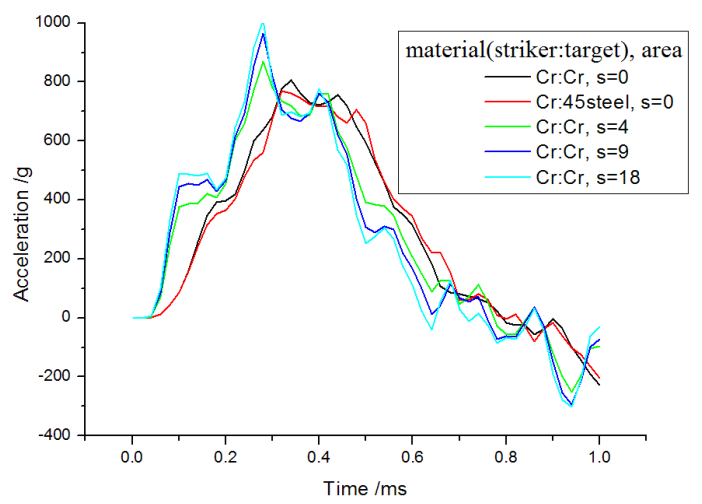

Figure 12: Acceleration response curves of numerical simulations at different initial conditions.

\section{Conclusions}

In this paper the dynamic response characteristics of the pendulum impact testing machine have been discussed. The paper consists of experimental results and numerical simulation results, and corresponding contrastive analysis. The acceleration response curves of their positions have been carefully analyzed at various initial conditions, concluding different geometrical configuration of striker and material properties of target. It points out the test results will not accuracy as the service conditions changes. The dynamic response characteristics analysis of the equipment indicates the methods for adjusting experimental results and how to calibrate the standard equipment. Those analyses are helpful for correcting experimental results, and even improving similar equipment quality in practical production.

\section{Acknowledgement}

This work was accomplished in the course of research sponsored by National Natural Science Foundation of China under the contract No. 11302211.

\section{References}

[1] F.J. Zhang, P. He, Z.F. Deng, G. Chen. The Test Reports of Inert Unit Impact Machine. Institute of Systems Engineering, CAEP, 2010.

[2] Wang Jun-feng, Zhang Zhi-yi. Impact Analysis of Impact Test Machine Base on LS-DYNA. Noise and Vibration Control, No. 2, 2007, December.

[3] Wu Hui, Zhang Cheng. Means of impact test and application of vibrationimpact platform. Ship Science and Technology, Vol. 34, No. 2, 2012.

[4] Xu Gang, Yu Zhihui. The drop-test facility with equipressure Style buffer. Test Technology and Testing Machine. Vol. 42, Nos. 1\&2. 
[5] W.Y. Li, Mechanical Properties Quick Manual of Steel [M]. China Machine Press, 2007, February.

[6] Z.M. Zeng. Mechanical Engineering Materials Handbook [M], China Machine Press, 2009, March. 